\title{
Ageing and learning disability ${ }^{\dagger}$
}

\author{
A. J. HOLLAND
}

\author{
Background Ageing is a continuation \\ of the developmental process and is \\ influenced by genetic and other biological \\ factors as well as personal and social \\ circumstances.
}

\begin{abstract}
Aims To identify some key biological, psychological and social issues relevant to how ageing might particularly effect people with learning disabilities.
\end{abstract}

Method This selected review considers the extent to which there are similarities and differences relative to people without learning disabilities.

Results There is a convergence, in later life, between people with a learning disability and those without, owing to the reduced life expectancy of people with more severe disabilities. People with Down's syndrome have particular risks of age-related problems relatively early in life.

\section{Conclusions The improved life expectancy of people with learning disabilities is well established. There is a lack of a concerted response to ensure that the best possible health and social care is provided for people with learning disabilities in later life.}

\section{Declaration of interest None.}

The changing age structure of the population identified in childhood as having a learning disability imposes important challenges. These include ensuring that the development of additional age-related disabilities can be prevented and maintaining general wellbeing and quality of life. This issue has arisen now because of significant changes in the care of people with learning disabilities and their improved life expectancy. 'Ageing' should be considered within the context of demographic changes in the population as a whole, as some of these health and social issues will be directly relevant to the older population of people with learning disabilities. From a research perspective, the question of whether the biological process of ageing and its social and emotional consequences differ for those with a learning disability needs investigation.

\section{AGEING: AN \\ EPIDEMIOLOGICAL PERSPECTIVE}

In the general population the importance of 'ageing', the potential for the development of additional disability and the economic impact of these factors have received considerable attention. There is no obvious and universally accepted definition of 'ageing', and no accepted theory as to the significant aetiological factors. Ageing is not synonymous with life span (or life expectancy) but is clearly intimately related to it. What has received increasing attention is the concept of disability-free life expectancy. Here the relationship between the process of 'ageing' and the associated risk of illness and disability on the one hand and increases in life span on the other is critical. The key question is whether increases in life span are inevitably associated with a greater number of years of disability in later life, or whether 'age-related disability' can be limited to the last few years of life (Robine \& Ritchie, 1991).
As life expectancy has improved and, in the developed world, birth rate has fallen, the proportion of older people has increased. One striking prediction is that the proportion of people over the age of 85 will increase from $6 \%$ of those aged over 60 to $11 \%$. In early old age there is a reasonably predictable pattern of cognitive change, but in this older elderly group the major debilitating disorders, such as Alzheimer's disease, become particularly prevalent and lead to the potential for a significant increase in disability among this group. Even in the developed world, there are differences between countries and between socio-economic classes in terms of life expectancy. For example, those living in southern Europe have among the best life expectancy. This pattern changes with migration and changes in lifestyle. Epidemiological studies suggest that, for example, diet and drinking habits have both positive and negative effects. From a different perspective there is also evidence that allelic variations at specific genetic loci (e.g. apolipoprotein $\varepsilon$ ) affect life expectancy and the propensity to age-related illnesses such as Alzheimer's disease (Rubinsztein, 1995). So, as life expectancy has improved for people with learning disabilities, the above influences, and most importantly the presence or absence of age-related disabilities, become increasingly relevant. Questions can appropriately be asked about the influence on the health and well-being of older people with learning disabilities of diet, the opportunity to smoke and the availability of health screening. We also need to investigate whether people with specific syndromes associated with learning disabilities are particularly prone to age-related problems and to dietary or other risk factors.

\section{Learning disability, ageing and life-expectancy}

When considering ageing and how it might affect people with learning disability, it is important to appreciate that this term refers to a highly heterogeneous group of people. These people all have evidence of delayed or abnormal early development together with significant intellectual and functional impairments, but they may differ markedly in terms of the cause, developmental profile, nature and extent of the impairments - and like all of us, in their personalities and social backgrounds. While a history of delayed or abnormal 
childhood development is essential if the label of 'learning disability' is to be applied, this process of 'development' should be considered to be lifelong and therefore continuing into later life. At the most basic level, severe and profound abnormalities of early development have a marked impact on life expectancy and on age-specific mortality rates. McGuigan et al (1995) discussed the complexities of investigating life expectancy among people with learning disabilities. In their analysis of data taken from learning disability registers covering three London boroughs and including births from 1896, they calculated standardised mortality ratios (SMRs; the number of observed deaths divided by the number of expected deaths) for the years 1982-1990. The SMRs were found to be above unity for both men and women with learning disabilities; in some groups they were significantly greater. The main limitation of the study was that the numbers were insufficient to allow classification according to severity or cause of disability. The cause of the learning disability is particularly relevant, because one of the groups most studied is people with Down's syndrome. In this syndrome the presence of trisomy 21 not only affects the early developmental profile of the child but is also associated with apparent premature ageing and an increased risk for developing Alzheimer's disease much earlier in life when compared with people with other causes of learning disability or with the general population. At biological, psychological and social levels such observations raise profound questions about the process of ageing, and the links between disorders of childhood development and later life - and, most importantly, about the extent to which people with learning disabilities have an increased morbidity and mortality, and why.

For the above reasons, the issues relating to ageing are more complex in the case of people with learning disabilities. While old age in the general population is clearly associated with increasing levels of disability, this is not, contrary to expectation, the case for people with learning disabilities as a group. This is because there are differential mortality rates depending on the severity of the learning disability. People with more severe learning disabilities still have a reduced life expectancy; therefore, across the spectrum of disability, there is a less severe level of learning disability in the group as a whole with increasing age (Moss, 1991). Table 1 (from Fryers, 1991) illustrates this. Age-specific prevalence rates of learning disability increase during early childhood as the extent of an individual's disability becomes apparent, but in later life they diminish because of the earlier death of those with more profound disabilities. The extent to which physical disabilities contribute to age-related morbidity in people with learning disability is well summarised in the review by Day $\&$ Jancar (1994). The studies they reviewed all report increasing prevalence of musculo-skeletal, cardiovascular, respiratory and neoplastic illnesses with age. However, in the case of some disorders (e.g. neoplasia), age-related increases in prevalence are relatively small. The studies did not clearly divide according to the level of learning disability.

Maaskant et al (1996) undertook a prospective cohort study of 1602 people resident in local facilities for people with learning disability, and looked for changes in care dependency over three years. They found what might have been predicted: that significant change over the period took place only in the younger group, who improved (people with Down's syndrome were excluded), and in those over 60 , who deteriorated in terms of care needs. These and other studies indicate that at an individual level increasing age after 60 brings with it the expected age-related impairments but, as described earlier, older people with learning disability when considered as a group have higher levels of functional abilities than the younger group (and lower levels

\section{Table I Estimates of age-specific prevalence of} severe intellectual impairment in an average English district of stable population in about 1990 (from Fryers, 1991, by permission of Oxford University Press)

\begin{tabular}{lc}
\hline Age (years) & $\begin{array}{c}\text { Prevalence } \\
\text { (per 1000 population) }\end{array}$ \\
\hline $0-4$ & $? 2.5$ \\
$5-9$ & 3.0 \\
$10-14$ & 4.0 \\
$15-19$ & 4.5 \\
$20-24$ & 5.0 \\
$25-34$ & 4.0 \\
$35-44$ & 3.0 \\
$45-54$ & 2.5 \\
$55-64$ & 2.0 \\
$65-74$ & 1.0 \\
$75+$ & very few \\
\hline
\end{tabular}

of challenging behaviour) (Moss, 1991). In Maaskant et al's (1996) study, rates of epilepsy were shown to be reduced in the older people with learning disabilities when compared with the younger ones. Importantly, however, sensory impairments characteristically associated with later life are found to a greater extent in those people with learning disabilities aged over 65 (Janicki et al, 1985; Evenhuis, 1995a,b). The various studies indicate that in later life there is a convergence in terms of health and social care needs between people with learning disability and the general population.

However, the study of age-related changes in older people with learning disabilities is complicated by the potential for very significant cohort effects; care therefore has to be taken when extrapolating from present-day findings to the future. For example, mortality rates and educational opportunities were very different 60 years ago than they are today. Infant mortality would have been higher and neonatal care unknown. In particular, those people with more severe learning disabilities who survived childhood are likely to have been self-selected on the basis of their ability to survive despite their disability, and may well therefore have carried with them into adult life a robustness, the effects of which may continue into old age. However, for most there would have been a much greater chance of being placed into an institutional setting, while many may not have received formal education, which did not have to be provided until the passing of the 1971 Education Act.

To summarise, the main biological and functional aspects of ageing are as follows:

(a) for people with learning disability as a group, functional ability improves in later life because of differential mortality rates leading to a shorter life expectancy for people with more severe learning disabilities and for people with Down's syndrome;

(b) age-related functional decline is observed in people with learning disabilities in later life, as it is in the general population;

(c) care needs to be taken in extrapolating from present studies of older people with learning disabilities because of the potential cohort effects due to changing patterns of childhood survival and improvements over the past 50 or so years in educational and other opportunities available to younger people. 


\section{AGEING AND THE MENTAL HEALTH OF PEOPLE WITH LEARNING DISABILITY}

While the detection and diagnosis of mental disorders affecting people with learning disabilities can be problematic because of the potential for impaired language development and the resulting difficulties in obtaining information on a person's mental state, it is clear that there are high rates of specific psychiatric and behavioural disorders. Patel et al (1993), in one of the first studies to use the Psychiatric Assessment Schedule for Adults with a Developmental Disability (PASADD), investigated the prevalence rate of psychiatric disorder in a cohort of people with learning disabilities aged 50 years or more living in one health district. Of the 105 participants, $12 \%$ had psychiatric disorders, and a further $12 \%$ had dementia. The disorders identified were mainly depression and anxiety. The authors commented that few of the sufferers were known to health or social services. In a more recent study (Cooper, 1997), 134 people with learning disabilities aged 65 years or more living in one geographical area were assessed. A specially constructed psychiatric interview was used, and rates of psychiatric disorder of over $65 \%$ were found (compared with $47 \%$ in the younger group). A large group was classified under the label 'behaviour disorder', but there were also significant rates of depression, anxiety and dementia. The author suggests a number of factors that might have accounted for the high rate. However, as she points out, most factors were common to both younger and older groups. Given that the psychiatric disorders seen in excess in the older groups were those found in the general elderly population, it is likely that the ageing process per se and associated social changes are the most significant influences. The importance of the above two studies is that they used sound diagnostic methods to identify important causes of age-related morbidity. Clearly, the ageing process in people with learning disability brings changes in the rates of physical and mental health problems similar to those found in people without pre-existing developmental disabilities. As happens more generally in the field of learning disability, it appears that the identification and subsequent treatment of such disorders is often not taking place.

\section{DEMENTIA AND ITS DIAGNOSIS IN PEOPLE WITH LEARNING DISABILITY}

Both of the studies mentioned above also found significant rates of dementia. The detection and diagnosis of this disorder are particularly important, given its effect on cognitive and functional ability. Dementia is a disorder of later life which manifests itself in those areas of function that may already be impaired in people with learning disability. Furthermore, if people are leading undemanding lives, the presence of functional decline may well go unnoticed, and any occasionally observed change may be dismissed as being 'part of the learning disability'. This problem of 'diagnostic overshadowing', and of dismissing changes in behaviour, personality or ability that would be taken very seriously in a person without a learning disability, are particularly relevant with dementia. For this reason, an expert group was established under the auspices of the ageing special interest research group of the International Association for the Scientific Study of Intellectual Disabilities to examine the issue (see, e.g., Aylward et al, 1997; Zigman et al, 1997). These papers stress the potential difficulty of diagnosis and suggest that greater emphasis should be placed on personality and behaviour changes as possible diagnostic characteristics in association with evidence of functional change.

In a study of 101 people with learning disabilities aged 50 and over that found 12 people with dementia, the presence of dementia was found to be associated with additional physical health problems and a greater proneness to violence and behavioural problems (Moss \& Patel, 1997). The authors pointed out that it is not simply the cognitive decline that is leading to functional decline, but a combination of factors. Interventions need to be targeted not only on issues relating to the dementia but also on those relating to physical health and environment. Dementia is particularly relevant in people with Down's syndrome. This matter is covered in more detail below.

\section{THE SPECIAL CASE OF PEOPLE WITH DOWN'S SYNDROME}

People with Down's syndrome illustrate most vividly how life expectancy has changed for people with learning disability, and how the focus of research and service developments has shifted because of the recognition that age-related health problems of later life are of particular significance. At the beginning of the 20th century, mean life expectancy for those with Down's syndrome was less than 10 years, whereas it is now nearer 50 years. Even so, the fact that people with Down's syndrome were at risk for developing the changes in the brain (plaques and neurofibrillary tangles) characteristic of Alzheimer's disease was well recognised at that time (see Oliver $\&$ Holland, 1986, for review). More recently, the focus of research has been to investigate the extent to which cognitive decline and dementia occur. Many studies have now confirmed that age-related cognitive decline and dementia affecting people with Down's syndrome occur $30-40$ years earlier in life than in the general population. The pattern of cognitive change observed in a proportion of people with Down's syndrome is similar to the cognitive course of Alzheimer's disease in the general population - for example, memory is affected relatively early and language relatively late (Oliver et al, 1998). Age-specific rates of dementia begin to increase in the patient's 30 s from $1-2 \%$ to $40 \%$ in the 50 s (Holland et al, 1998). There is also some evidence that personality changes may precede more obvious cognitive changes in some people. With the onset of apparent changes in either behaviour or functional ability, a key task is to identify a possible cause. Importantly, other agerelated changes also occur and may mimic dementia; such changes include increasing sensory impairments and thyroid disorders (Prasher \& Chung, 1996).

Although it was recognised over 100 years ago that people with Down's syndrome may get a 'sort of precipitated senility', the understanding of this relationship was for a long time a largely neuropathological one. Numerous studies reported evidence of plaques and neurofibrillary tangles in people with Down's syndrome who had died in early adult life. Some studies retrospectively looked for evidence of decline and reported evidence of personality changes, functional decline and the presence of neurological dysfunction (Oliver \& Holland, 1986). This was followed by crosssectional clinical studies, and later by more robust data about the likely age-related prevalence of clinical dementia (i.e. Alzheimer's disease). The neuropathological studies seemed to indicate that everyone with Down's syndrome developed significant 
Alzheimer-like neuropathology as early as their 30s, yet as the clinical studies were undertaken it became apparent that there were people with Down's syndrome living into their 50s and 60s who were clearly not developing dementia. This apparent discrepancy is now well established and remains an important research issue. Clearly, there are factors which both increase and decrease the risk of developing Alzheimer's disease with age. The most striking is the influence of the Apo $\varepsilon$ alleles. The effect is the same as that found in the general population, with the Apo $\varepsilon 2$ protecting and the Apo $\varepsilon 4$ allele increasing the risk (Rubinsztein et al, 2000).

The role of amyloid and excess amyloid production appears to be very significant in understanding the increased risk for Alzheimer's disease in people with Down's syndrome. The discovery that the amyloid gene is localised on chromosome 21 came about because of the known association between Down's syndrome and Alzheimer's disease which suggested that chromosome 21 may be a candidate site for an Alzheimer's disease gene. It is also clear that there is diffuse cerebral amyloid deposition starting early in life and ultimately leading to plaques and tangle formation (Rumble et al, 1989; see also review by Mann, 1993). Given that not all people with Down's syndrome develop Alzheimer's disease it would seem that excess amyloid expression cannot by itself account for the high risk of developing Alzheimer's disease. Furthermore, people with Down's syndrome still have a reduced mean life expectancy compared with the general population (45 v. 75 years) and there has been no adequate explanation for that fact, although the effect of increased oxidative activity due to the presence of the superoxide dismutase gene on chromosome 21 or amyloid deposition leading to Alzheimer's disease and premature death are possible explanations. There remain important research questions that need to be addressed with respect to the link between Down's syndrome and Alzheimer's disease. The ultimate objective of such research is the development of treatments that prevent or at least delay the onset of dementia.

\section{SOCIAL ASPECTS OF AGEING}

Ageing is, of course, associated with more than just biological changes, cognitive decline and increasing risk of physical and psychiatric disorders. It is also associated with significant social and economic changes. It is perhaps in this aspect of ageing that there are some of the biggest contrasts between those with and without learning disabilities. First, for most of the population life is structured into infancy, childhood, working adult life and retirement. For people with learning disabilities, many of the expectations that people have of life are not available. The most striking example is work. Although supported employment schemes and more meaningful daytime occupation are becoming available to people with learning disabilities, provision in this area remains seriously deficient. Without work or its equivalent there can be no retirement. These issues were well illustrated by Ashman et al (1995), who investigated the employment and retirement status of people with learning disabilities aged over 55 living in two Australian states. Many of those interviewed wanted to work or to have worked, but the majority had never had the opportunity. Another problem concerning retirement is that either the resourcing of group homes forces people to go to a day centre during the day (and the option of retiring and spending one's weekdays at home is not available) or if people are at home very little social activity is available to them. In Ashman et al's study the average number of weekly social activities for those people with learning disability who had retired was 2.7 .

Also important are the ageing and death of family members and the life histories of families. Increasing age for people with learning disabilities is associated with the loss not only of family members but possibly also information about the person him- or herself. If a person has limited language, how can he or she know about his or her past, likes and dislikes, wishes, etc.? A key aspect of ageing is the preservation of that knowledge, perhaps through the use of life story books and other means. The need for preparation for bereavement and for support after it in people with learning disability is now recognised and can be undertaken with the help of specially prepared books. A study by Hollins \& Esterhuyzen (1997) of 50 people who had experienced the death of a parent reported high rates of subsequent behavioural disturbance and emotional distress which were often not recognised as being in response to the loss, indicating that in many cases bereavement is still not taken seriously.
The social aspects of ageing are summarised as follows:

(a) differences in the overall structure of the life of a person with learning disabilities and the funding of social care lead to a failure to recognise and plan for retirement and to provide the necessary change in lifestyle when required;

(b) ageing is associated not only with the increasing likelihood of the death of family members but also with the potential for the loss of knowledge about the past experiences of the person with learning disability, especially if he or she is unable to relate it for him or herself (life story books are one way of trying to maintain that knowledge);

(c) as with the population generally, the experience of bereavement by people with learning disabilities can be associated with considerable behavioural and emotional changes that can go unrecognised, resulting in the person failing to receive appropriate support.

\section{SERVICES}

In a detailed paper for the Mental Health Foundation, Hogg \& Lambe (1998) reviewed the published literature on residential services and family care-giving for older people with learning disabilities. They pointed out that the numbers involved are not great. They emphasise that, at present, services for people with learning disability remain inherently specialist and are predominantly segregated from the mainstream. They point out that learning disability services remain unprepared for the changing needs of older people with learning disability and that generic services for the elderly are not readily accessible. The 'segregation $v$. integration' debate clearly applies to older people. There are competing arguments that may to some extent depend upon local and national differences in the funding and provision for both the elderly and people with learning disability. Much of the work on service provision for older people with learning disabilities has been undertaken in the USA (Janicki et al, 1995). For reasons of both non-discrimination and funding, Janicki et al advocated the integration of services for the elderly, and reported considerable success. There is clearly a difficult balance to be found between supporting people so that they may remain in their 
homes despite increasing infirmity and the need for different types of support and staff skills. In the UK, a further concern is that services for the elderly may be less well resourced than social care services for people with learning disabilities, and if so there will be a decline in quality of life when a transition between these services is made.

The key health and social care service requirements of older people with learning disabilities are summarised as follows:

(a) the social care environment should be responsive to the changing lifestyles and social circumstances of later life and acknowledge, when appropriate, the place of retirement; it should also look to establishing links with other services for older people in order to meet the individual's changing needs;

(b) health professionals familiar with agerelated health problems and the diagnosis, treatment and management of the health problems specific to later life (e.g. dementia) should be available; services should include general practice support, mainstream health services (e.g. for assessment of sensory impairments) and additional specialist learning disability and elderly services;

(c) carers and health and social care providers should acknowledge the need for emotional support for people with learning disability whose family circumstances change (e.g. through bereavement) and recognise the importance of helping people with learning disability to retain knowledge of their past and of their families.

In this paper it has been argued that with increasing age there is a convergence in terms of health and social care needs between those with and without disability. In childhood and early adult life, specialist services provide for the health and social care needs of people with learning disabilities. In later life the additional dimension of age-related health problems and the changing social care perspective, in which retirement and leisure take priority over paid employment, become apparent. How then do we resolve such tensions? A report from the Social Services Inspectorate (Harris, 1997) emphasised the importance of strategic planning, a flexible funding system that can accommodate change, close collaboration with other agencies and the availability of health expertise - in short, informed and individualised needs-led assessments and care planning.

\section{CLINICAL IMPLICATIONS}

- Health and social services need to develop joint strategies for ensuring that the changing needs of older people with learning disabilities can be met.

- Further research is required to investigate the influences on age-related morbidity and mortality, particularly in those with more severe learning disabilities or Down's syndrome.

- The maintenance of the physical and mental health of people with learning disabilities and the early detection and treatment of both physical and mental health problems are key responsibilities for primary and specialist health services.

\section{LIMITATIONS}

- A full understanding requires the integration of biological, psychological and social research from both the ageing and learning disability literature; this has not been achieved sufficiently.

- This review is selective and, given the limitations of available research, the conclusions have been influenced by personal experience and by work in local services; they have not therefore been determined in a completely objective manner.

- The review has raised more questions than it has answered.

A. J. HOLLAND, MRCPsych, Section of Developmental Psychiatry, University of Cambridge, Douglas House, I8b Trumpington Road, Cambridge CB2 $2 \mathrm{AH}$

(First received 12 July 1999, final revision 17 September 1999, accepted 21 September 1999)

\section{ACKNOWLEDGEMENTS}

I thank Robbie Patterson for her administrative and secretarial support and Bonnie Kemske for reading the preliminary manuscript.

\section{REFERENCES}

Ashman, A. F., Suttie, J. N. \& Bramley, J. (1995)

Employment, retirement and elderly persons with an intellectual disability. Journal of Intellectual Disability Research, 39, 107-115.

Aylward, E., Burt, D., Thorpe, L., et al (1997) Diagnosis of dementia in individuals with intellectual disability: report of the task force for development of criteria for diagnosis of dementia in individuals with mental retardation. Journal of Intellectual Disability Research, 4I, 152-164.

Cooper, S.-A. (1997) Epidemiology of psychiatric disorders in elderly compared with younger adults with learning disabilities. British Journal of Psychiatry, $\mathbf{1 7 0}$ 375-380.

Day, K. \& Jancar, J. (1994) Mental and physical health in mental handicap: a review. Journal of Intellectual Disability Research, 38, 257-264.

Evenhuis, H. M. (1995a) Medical aspects of ageing in a population with intellectual disability: I. Visual impairment. Journal of Intellectual Disability Research, 39, 19-25.
- (1995b) Medical aspects of ageing in a population with intellectual disability: II. Hearing impairment. Journal of Intellectual Disability Research, 39, 27-33.

Fryers, T. (1991) Public health approaches to mental retardation: handicap due to intellectual impairment. In Oxford Textbook of Public Health (eds W.W. Holland, R. Detels \& G. Knox), pp. 485-508. Oxford: Oxford University Press.

Harris, J. (1997) Services for Older People with Learning Disabilities. London: Social Service Inspectorate, Department of Health.

Hogg, J. \& Lambe, L. (1998) Older People with Learning Disabilities: A Review of the Literature of Residential Services and Family Caregiving. Dundee: White Top Research Unit, University of Dundee.

Holland, A. J., Hon, J., Huppert, F. A., et al (1998)

Population-based study of the prevalence and presentation of dementia in adults with Down's syndrome. British Journal of Psychiatry, I72, 493-498.

Hollins, S. \& Esterhuyzen, A. (1997) Bereavement and grief in adults with learning disabilities. British Journal of Psychiatry, 170, 497-501.

Janicki, M. P., Ackerman, L. \& Jacobson, W. (1985)

State developmental disabilities/ageing plans and planning for an older developmentally disabled population. Mental Retardation, 23, 297-301.

_, Heller, T., Seltzer, G., et al (1995) Practice

Guidelines for the Clinical Assessment and Care

Management of Alzheimer and other Dementias among 
Adults with Mental Retardation. Washington, DC: American Association on Mental Retardation.

Maaskant, M. A., van den Akker, M., Kessels, A. G. H., et al (1996) Care dependence and activities of daily living in relation to ageing: results of a longitudinal study. Journal of Intellectual Disability Research, 40, 535-543.

Mann, D. M. A. (1993) Association between Alzheimer disease and Down syndrome: neuropathological observation. In Alzheimer Disease, Down Syndrome and their Relationship (eds J. M. Berg, H. Karlinsky \& A. J. Holland), pp. 71-92. Oxford: Oxford University Press.

McGuigan, S. M., Hollins, S. \& Attard, M. (1995) Age-specific standardized mortality rates in people with learning disability. Journal of Intellectual Disability Research, 39, 527-531.

Moss, S. C. (1991) Age and functional abilities of people with a mental handicap: evidence from the Wessex Mental Handicap Register. Journal of Mental Deficiency Research, 35, 430-445.
_ \& Patel, P. (1997) Dementia in older people with intellectual disability: symptoms of physical and mental illness, and levels of adaptive behaviour. Journal of Intellectual Disability Research, 4I, 60-69.

Oliver, C. \& Holland, A. J. (1986) Down's syndrome and Alzheimer's disease: a review. Psychological Medicine, 16, 307-322.

_ , Crayton, L., Holland, A., et al (1998) A four year prospective study of age related cognitive change in adults with Down's syndrome. Psychological Medicine, 28, 1365-1377.

Patel, D., Goldberg, D. \& Moss, S. (1993) Psychiatric morbidity in older people with moderate and severe learning disability. II: The prevalence study. British Journal of Psychiatry, 163, 48I-49I.

Prasher, V. P. \& Chung, M. C. (1996) Causes of age related decline in adaptive behavior of adults with Down syndrome: differential diagnoses of dementia. American Journal of Mental Retardation, 10I, 175-183.
Robine, J. M. \& Ritchie, K. (1991) Healthy life expectancy: evaluation of global indicator of change in population health. British Medical Journal, 302, 457-460.

Rubinsztein, D. C. (1995) Apolipoprotein E: a review of its roles in lipoprotein metabolism, neuronal growth and repair and as a risk factor for Alzheimer's disease. Psychological Medicine, 25, 223-229.

_ , Hon, J., Stevens, F., et al (2000) ApoE genotype and risk of dementia in Down's syndrome. Neuropsychiatric Genetics, in press.

\section{Rumble, B., Retallack, R., Hilbich, C., et al (1989)} Amyloid A4 protein and its precursor in Down's syndrome and Alzheimer's disease. New England Journal of Medicine, 320, 1446-1452.

Zigman, W., Schupf, N., Haveman, M., et al (1997) The epidemiology of Alzheimer disease in intellectual disability; results and recommendations from an international conference. Journal of Intellectual Disability Research, 4I, 76-80. 